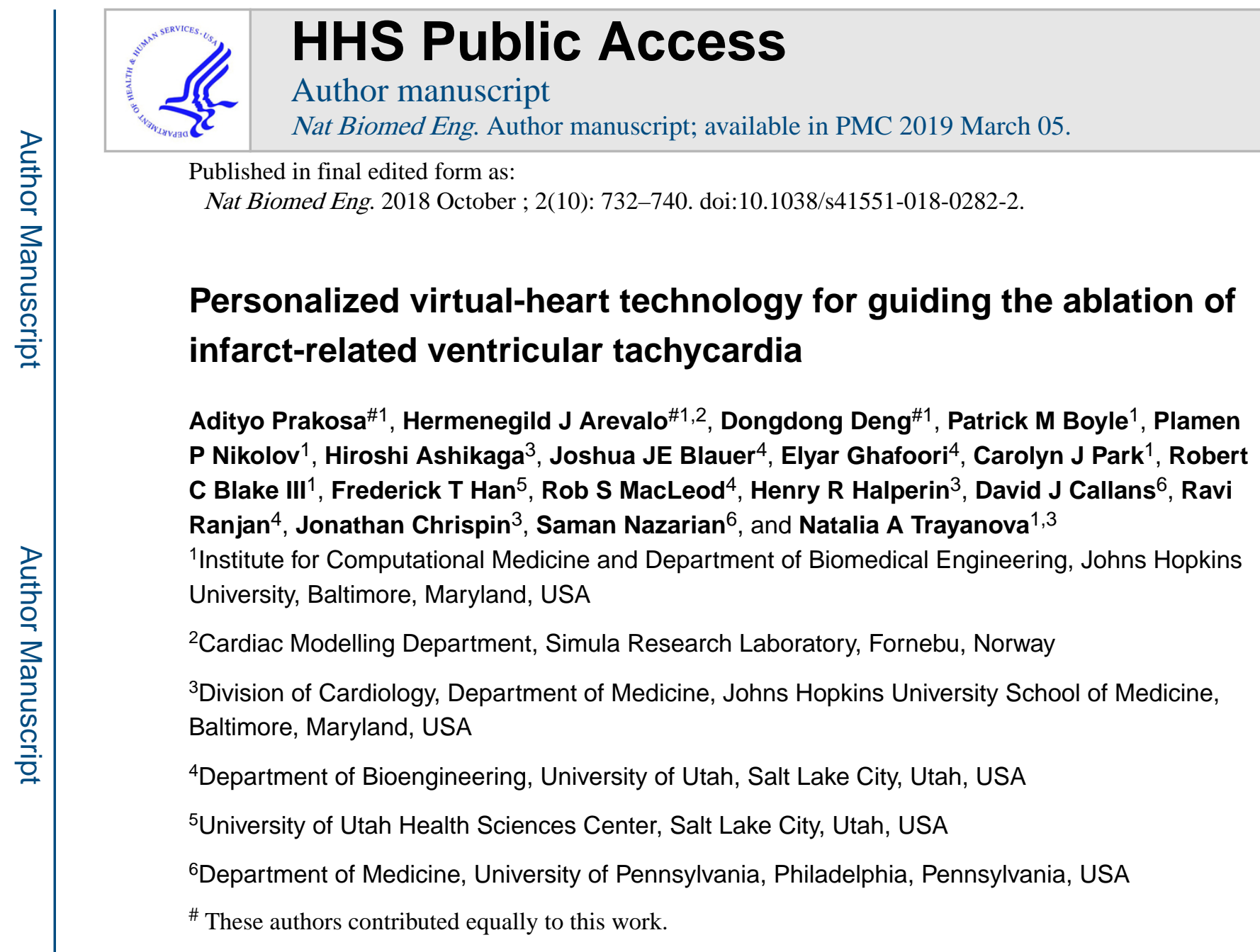

\title{
Abstract
}

Ventricular tachycardia (VT), which can lead to sudden cardiac death, occurs frequently in patients with myocardial infarction. Catheter-based radiofrequency ablation of cardiac tissue has achieved only modest efficacy, owing to the inaccurate identification of ablation targets by current electrical mapping techniques, which can lead to extensive lesions and to a prolonged, poorly tolerated

Users may view, print, copy, and download text and data-mine the content in such documents, for the purposes of academic research, subject always to the full Conditions of use: http://www.nature.com/authors/editorial_policies/license.html\#terms

Corresponding Author: Natalia A Trayanova, PhD, Institute for Computational Medicine, Johns Hopkins University, $3400 \mathrm{~N}$. Charles St, 208 Hackerman Hall, Baltimore, MD 21218, Phone: (410) 516-4375, Fax: (410) 516-5294, ntrayanova@ jhu.edu. Author Contributions. AP, HJA, DD, HA, and PPN performed animal and human LGE-MRI scan segmentation and model creation. AP, HJA, DD and NAT designed the simulation protocols. DD, HJA, AP and PN performed simulations of VT in all models. DD, AP, PMB, HJA, and NAT analyzed the data. AP developed the pipeline for model generation from MRI scans with ICD artifact. DD and AP adapted the automatic algorithm for determining the ablation tragets in the ventricles. HH provided the swine MRI and electrophysiological data, as well as input for the animal study. SN provided part of the human MRI scans (at Johns Hopkins), conducted the prospectove studies at University of Pennsylvania, and provided clinical guidance and input. JC provided the reminder of the human MRI scans and patient outcomes. AP developed the methodology for input of simulation data into the clinical CARTO mapping system. JB, EG, RM, and RR developed and implemented the clinical protocols at the University of Utah. RR and FH recruited patients and conducted VT ablations for the prospective human study at the University of Utah. SN and DC recruited patients and conducted VT ablations for the prospective human study at the University of Pennsylvania. NAT initiated the collaborations, designed and coordinated the studies with contributions from: HJA and HH (retroprospective swine and human studies), SN and RR (prospective human studies), and AP and SN (retrospective human with ICD study), and supervised all simulation studies. HJA, DD, AP, PMB, EG, and RR generated figures, tables, and movies. NAT wrote the manuscript with input from AP and HJA. All authors discussed the results and commented on the manuscript.

Competing Interests. NAT holds partial ownership of CardioSolv Ablation Technologies LLC. SN is a scientific advisor to CardioSolv Ablation Technologies LLC. The other authors declare no competing financial and non-financial interests.

Additional Information. Reprints and permissions information are available at www.nature.com/reprints. Correspondence and requests for materials should be addressed to NAT (ntrayanova@jhu.edu). 
procedure. Here we show that personalized virtual-heart technology based on cardiac imaging and computational modelling can identify optimal infarct-related VT ablation targets in retrospective animal ( 5 swine) and human studies (21 patients) and in a prospective feasibility study (5 patients). We first assessed in retrospective studies (one of which included a proportion of clinical images with artifacts) the capability of the technology to determine the minimum-size ablation targets for eradicating all VTs. In the prospective study, VT sites predicted by the technology were targeted directly, without relying on prior electrical mapping. The approach could improve infarctrelated VT ablation guidance, where accurate identification of patient-specific optimal targets could be achieved on a personalized virtual heart prior to the clinical procedure.

Ventricular tachycardia (VT), a life-threatening fast heart rhythm, occurs frequently in patients with myocardial infarction, can lead to sudden cardiac death. Catheter-based radiofrequency ablation, which delivers energy to destroy the ability of cardiac tissue to conduct electrical signals, offers the possibility of permanent cure, as it disrupts the propagation of abnormal electrical waves sustaining VT. Eliminating infarct-related VT with ablation has achieved, however, only modest success, $50-88 \% \%^{1,2}$. This stems from limitations in current techniques for mapping the electrical functioning of the heart and identifying the targets for ablation. These include limited spatial sampling during mapping, with resolution often insufficient to identify critical VT propagation pathways ${ }^{3-5}$, hemodynamic intolerance during the VT ablation procedure, and ambiguities in correlating electrical maps with heart anatomy ${ }^{6}$. Furthermore, the complex three-dimensional pathways of cardiac impulse propagation around/through the zone of infarct during VT are often difficult to reconstruct by mapping the ventricular surfaces only 7,8 . These limitations could translate into inaccurate ablation targets and extensive lesions, and into prolonged duration of the procedure (4-12 hours), potentially increasing the risk of complications and radiation overexposure $^{9,10}$.

Here we present the proof-of-concept of a "virtual-heart" methodology for determining the optimal targets for infarct-related VT ablation that completely eliminates the need for invasive electrical mapping. We term this non-invasive approach VAAT, Virtual-heart Arrhythmia Ablation Targeting. The approach is based on cardiac imaging and computational modeling, and is personalized to each patient. Similar to our Virtual-heart Arrhythmia Risk Prediction (VARP) approach ${ }^{11}$ for stratifying patients at risk of sudden cardiac death developed previously, the methodology involves constructing threedimensional computer models of patients' hearts (ventricles) from clinical magnetic resonance imaging (MRI) data and executing simulations with to evaluate the patientspecific VTs. In the current study, the virtual-heart simulation strategy is further developed and applied in a different clinical arena: the non-invasive planning and guidance of the clinical procedure of VT ablation. In the VAAT approach, once all patient-specific VTs are evaluated, we determine automatically, using an algorithm, the optimal ablation targets that render, with minimum lesion size, each heart not inducible for VT from any ectopic site. In a significant departure from all current VT ablation procedures, the VAAT approach targets termination not only of VTs that are clinically manifested or induced at the time of procedure, but of all VTs that could arise from the given post-infarction substrate, including those that might arise following initial ablation, thus potentially eliminating the need for 
repeated ablations and offering long-term freedom from VT. In addition to determining automatically the ablation targets, VAAT overcomes significant additional technical challenges in combining virtual-heart output with the clinical electroanatomical navigation system, and represents the first direct integration of computational modelling in cardiac patient care.

Presented below are animal $(\mathrm{n}=5)$ and human $(\mathrm{n}=21)$ retrospective studies, and a proof-ofconcept prospective patient study ( $\mathrm{n}=5$ ), illustrating the utility of VAAT to non-invasively determine the optimal ablation targets.

\section{Overview of VAAT}

The arrowed steps in the flowchart of Fig. 1a summarize the VAAT method, as we envision it for guidance of the clinical procedure of infarct-related VT ablation. First, an individualized geometric model of the post-infarction ventricles is reconstructed from lategadolinium-enhanced (LGE) MRI, as previously described ${ }^{11,12}$, with representations of both scar and infarct border zone (termed also gray zone, GZ). Determining the personalized fiber orientations using a validated approach ${ }^{13}$ completes geometric reconstruction. Regionspecific cell and tissue electrical properties are then assigned to the geometric model. To determine all VT reentrant pathways that the infarct-remodeled ventricular substrate can sustain, we conduct a virtual multi-site delivery of electrical stimuli (pacing) from a number of bi-ventricular locations, each attempting to elicit VT from a site positioned differently with respect to the infarct. The methodology for evaluating the VTs in post-infarction heart models has been previously presented and validated in an arrhythmia risk prediction clinical study involving 41 patients ${ }^{11}$.

As a next step in the VAAT method, in each personalized virtual heart we determine the minimum-size (i.e. optimal) ablation lesions that render it no longer inducible for VT from any pacing location. These optimal targets are determined using an automatic algorithm we developed recently ${ }^{14}$. The algorithm represents reentrant wave propagation associated with each of the inducible VTs in the given heart model as a flow network, and identifies the smallest amount of tissue that, when eliminated from the network, disrupts and terminates the flow. The algorithm was validated in a retrospective study in patients with atypical atrial flutter ${ }^{14}$, and this is its first application to ventricular arrhythmias.

Once the VAAT ablation lesions are calculated, they are incorporated back in the corresponding virtual-heart model and the VT inducibility protocol is repeated to assess whether VT is inducible from any of the pacing sites. Should a new VT arise in the modified-by-ablation ventricular substrate, the VAAT protocol is repeated until complete VT non-inducibility is achieved. The resulting set of ablation lesions represents the targets that will be directly approached during the clinical procedure, without any electrical mapping. The set of VAAT ablation lesions is expected to result in the complete elimination of the ability of the infarct-remodeled ventricular substrate to sustain VT.

In the prospective study, VAAT is executed during the brief time interval ( $<24$ hours) between the acquisition of patient MRI and the clinical ablation procedure. Once calculated, 
the set of predicted ablation targets are exported, then uploaded and co-registered in the clinical electroanatomical navigation system before the procedure commences.

Full description of the VAAT methodology is provided in Methods.

\section{VAAT Proof-of-concept Studies}

The capability of VAAT to non-invasively determine the ablation targets that eliminate all infarct-related VTs with minimum lesions was illustrated in retrospective and prospective studies (rectangles in Fig. 1a).

A retrospective animal study (swine model, $\mathrm{n}=5$ ) showed that VAAT is able to reproduce successful mapping-based ablation outcomes but with reduced lesion size, and to provide superior targets when mapping-based ablation failed. For the two cases of successful experimental ablation (Swine 1\&2, Fig. $1 \mathrm{~b}$ top and Supplementary Fig. 1 top), VAAT lesions were in the same regions as experimental (red dots mark the ablation catheter tip, visible in computed tomography (CT) scans), highlighting the predictive capability of the virtual-heart approach. Analysis of post-ablation MRI scans showed significant difference in lesion volumes between experiment and VAAT (Swine 1: $0.39 \mathrm{~cm}^{3}$ vs. $0.19 \mathrm{~cm}^{3}$; Swine 2: $0.37 \mathrm{~cm}^{3}$ vs. $0.28 \mathrm{~cm}^{3}$ ). In the remaining animals (Swine 3,4 , and 5 , Fig. $1 \mathrm{~b}$ bottom row and Supplementary Fig. 1), mapping-based lesions that failed to terminate VT were located away from the locations of the VAAT targets that eliminated the VTs (see Supplementary Table 1 for simulated lesion volumes).

Two retrospective human studies ( $\mathrm{n}=21$ total) in patients that had undergone pre-procedure LGE-MRI and successful infarct-related VT ablation were next undertaken to demonstrate that VAAT lesions fall within the areas ablated clinically. The first study was in patients without implantable cardioverter defibrillator (ICD) devices $(n=16)$. Four cases are shown in Fig. 2, another 10 are presented in Supplementary Fig. 2. Visual inspection of Fig. 2, rightmost panels, shows that in patients 2-4, the predicted lesions (see Supplementary Table 2 for calculated lesion sizes in all 16 patients) were smaller than the clinical (compare to extent of endocardial surface enclosing ablation catheter tip locations in the electroanatomical navigation, i.e. CARTO, maps; each lesion is of typical size ${ }^{15} 6.7 \mathrm{~mm} \times 9.4 \mathrm{~mm} \times 3.4 \mathrm{~mm}$, i.e. $\left.0.21 \mathrm{~cm}^{3}\right)$.

Since majority of patients who undergo infarct-related VT ablation nowadays have ICD, which imposes image artifact, we conducted a second human retrospective study ( $n=5)$ to asses feasibility of determining the optimal infarct-related VT ablation targets in patients with ICDs despite the artifact burden, thus broadening the clinical utility of the VAAT approach. For this purpose, we developed an enhanced model generation methodology (see Methods) applicable for cases where the ICD artifact did not cover the LGE regions. Of the 5 randomly selected patients with ICDs, two had a negligible myocardial artifact burden, while in the remaining three the artifact ranged from 46 to $62 \%$. LGE regions indicative of infarct-related structural remodeling were identifiable outside of the ICD artifact in all 5 cases. In this retrospective study, simulations were conducted blind to the clinical data. For all patients, targets predicted by VAAT generally corresponded to clinical lesions (Fig.4 
presents the ablation results for two patients, with Supplementary Movie 1 showing VT in one; the rest are shown in Supplementary Fig. 4), but were smaller in size; myocardial burden artifacts and simulated lesion sizes are presented in Supplementary Table 3). Note that in Patient 1, the clinical ablation lesion known in this case to have resulted in acute VT termination and the VAAT lesion fully coincided. In this patient's clinical procedure, the infarct scar was additionally encircled with lesions targeting the GZ in an attempt to prevent VT recurrence; VAAT demonstrated that this lesion was sufficient to eliminate the arrhythmogenic propensity of infarct substrate. This retrospective patient study showed that despite the ICD artifact, in cases when the ICD artifact does not obscure the zone of infarct, the VAAT approach could non-invasively predict the optimal VT ablation targets and thus be used to guide clinical ablation

We next embarked on a prospective human study $(\mathrm{n}=5)$, at two different clinical centers, University of Utah (3 patients, time period January 2015 - December 2016) and University of Pennsylvania (2 patients, time period November-December 2017), to assess the feasibility of VT ablation that directly targets sites predicted by the VAAT approach, without prior intracardiac mapping. The prospective study also served to determine whether the VAAT protocol could be executed within the relevant clinical timeframe.

Fig. 3 presents two cases, one from each clinical center, of successful VAAT-guided ablations in a post-infarction patients with inducible sustained VT. Model-generated ventricular images with the VAAT targets were imported into the clinical electroanatomical navigation system (Fig. 3), so that the ablation catheter could be directly steered towards the targets. After ablation of the VAAT targets, a clinical VT inducibility pacing protocol (see Methods for clinical protocol detail) was executed to confirm that VT was no longer inducible. Activation maps of the two VT morphologies in a Utah patient are shown in Fig. 3b, while Supplementary Fig. 3 and Supplementary Movie 2 present transmembrane potential maps over time for one of the two induced VT morphologies in this patient. The patient has remained VT-free since, over the 23-months follow-up period. Similarly, in another patient ablated the same center, VT was also inducible, and the VAAT-predicted sites were directly ablated. However, the patient went into ventricular fibrillation following the procedure and had to be cardioverted. Nonetheless, following the VAAT-driven ablation, the patient has remained VT-free since, over the follow-up period of 21 months. In the third patient, despite the presence of scar on the MRI, the model predicted that VT would not be inducible. This matched the clinical outcome, as infarct-related VT was indeed not inducible during the electrophysiological study despite the patient's history of myocardial infarction; instead, the sustained wide complex tachycardia was found to be supra-ventricular tachycardia with aberrancy.

Among the two prospective patients ablated at the University of Pennsylvania, one was not inducible for VT in the clinic. VAAT predicted VT non-inducibility prior to the clinical procedure; the model was not inducible for VT despite the presence of scar/GZ. In the other patient, the VAAT targets were successfully ablated, and VT terminated. Two VT morphologies in this patient are shown in Fig. 3f, with targets in Fig.3g, and co-registration of VAAT targets and ablation catheter tip locations during the procedure (Fig. 3h). The prospective human studies demonstrated that a simulation-driven VT ablation procedure is 
feasible within the clinical workflow and timeline, and that it can be executed at different clinical centers.

\section{DISCUSSION}

This study presents VAAT, a virtual-heart approach to predict the optimal targets for infarctrelated VT ablation. The approach could eliminate the need for invasive electrical mapping during the clinical procedure. VAAT determines the ablation targets non-invasively, on the basis of a comprehensive evaluation of all three-dimensional VT reentrant circuits that the infarct-remodeled substrate can support. The targets are calculated by an algorithm that determines the minimum amount of tissue which, when rendered non-excitable, eliminates all VTs, and not only those manifested clinically or induced during the procedure, including those that could arise if the arrhythmogenic propensity of the substrate was not fully eliminated during the initial ablation. The VAAT approach thus have the potential to not only guide infarct-related VT without prior electrical mapping, but to also eliminate the need for repeated ablations, offering long-term freedom from VT.

Here we describe the conceptual underpinning of the approach, and present comparisons of VAAT lesions with retrospective experimental (5 animals) and clinical (21 patients) ablation lesion data. As the lesion data are retrospective, and represents information about all lesions inflicted during the clinical procedure, such a comparison serves to primarily demonstrate VAAT lesion location correspondence and lesion size advantage. The promising results from the proof-of-concept prospective studies (5 patients) at two clinical centers demonstrate the feasibility of conducting a large prospective patient study, and underscore the VAAT potential for translation to the clinic for guidance of VT ablation in patients. Should the capability of the VAAT approach be demonstrated in such prospective studies, VAAT holds the promise to radically change infarct-related VT ablation, engendering swift and precise delivery of ablation without prior electrical mapping, and eradicating all possible VTs the substrate can sustain, thus eliminating or at least reducing the need for redo procedures. This could result in a dramatic improvement in the efficacy of and tolerance for the therapy, as well as in reduction of post-procedure complications. Finally, since early use of VT ablation post-infarction has been shown to result in much improved patient outcomes (SMASH-VT Trial) ${ }^{16}$, an accurate and easily executed VT ablation will lead to significant widening of the therapeutic potential of the procedure: the latter could become first line therapy for infarctrelated VT, in contrast to its current administering after drugs have failed.

Notably, the VAAT approach bypasses the need to establish the entity targeted by ablation, whether channels in the scar ${ }^{17,18}$, the $\mathrm{GZ}^{19}$, or reentrant drivers ${ }^{20}$. Additional utility of the approach is derived from the fact that it could inform the operator whether the approach to the target(s) is endo- or epicardial, as these require different anticoagulation setups, thus providing additional money and time savings, and decreasing radiation and associated complications. Importantly, since the predicted targets are three-dimensional, in some cases they may not be fully reachable via an endo- or epicardial approach; in such cases, we envision re-computing the targets, taking into account that a given portion of the initial predicted target will need to remain excitable. 
The VAAT approach could be extended ${ }^{21}$ to the rapidly increasing population of patients with non-ischemic cardiomyopathy, where myocardial structure incorporates distributed fibrosis/scar, and to patients with arrhythmias secondary to surgical repair in congenital heart disease, such as Tetralogy of Fallot. Furthermore, since VAAT is based on a simulation method representing processes from the molecular to the whole organ, it could be potentially modified to input patient-specific genetic and pathophysiological data, and thus its application could be broadened to arrhythmias arising from cardiac diseases of various etiologies. Integrating image-based computational modeling into treatments for heart rhythm disorders could thus advance personalized approaches to heart disease.

\section{METHODS}

\section{Retrospective Animal Study: MRI Datasets and Experimental Protocol}

The retrospective animal study (swine model, $\mathrm{n}=5$ ) was conducted to demonstrate that the VAAT methodology was able to reproduce successful mapping-based ablation outcomes but with reduced target size, and to provide superior alternative targets when mapping-based ablation failed. The details of both in vivo MRI acquisition and the electrophysiology study (EPS) in these animals have been previously described ${ }^{22}$. In brief, myocardial infarction was induced via occlusion of the mid-left anterior descending coronary artery. All animals were $\sim 2$ months old, with weight ranging from $18-23 \mathrm{~kg}$, and 2/5 were male. Four weeks after occlusion, high-resolution in vivo cardiac MRI (3.0-T scanner, Achieva, Philips Medical Systems, Best, The Netherlands) with late gadolinium enhancement (LGE) was acquired at a resolution of $976 \times 976 \times 4000 \mu \mathrm{m}^{3}$. A computed tomography (CT) scan was also acquired. One day after image acquisition, the animals underwent a traditional EPS with intracardiac mapping to determine the infarct-related VT targets and perform ablation guided by the CARTO XP electroanatomic mapping system (Biosense Webster, Inc; Diamond Bar, California). Seven to nine days post-ablation, a follow up EPS was conducted to determine if ablation resulted in VT non-inducibility. In two of the five swine hearts, ablation succeeded and VT remained non-inducible; in the other 3, ablation failed. A post-ablation in-vivo LGEMRI was also performed at that time in order to document actual extent of ablation lesions.

\section{Retrospective Patient Studies: Clinical MRI Datasets and Protocols}

Both human retrospective studies ( $\mathrm{n}=21$ total), with and without ICDs, included patients whose pre-ablation cardiac LGE-MRI showed myocardial scar (on a 1.5-T scanner, Avanto, Siemens Medical Solutions, Erlangen, Germany; resolution $\sim 1.5 \times 1.5 \times 10.2 \mathrm{~mm})$. All the patients underwent the standard EPS (i.e. voltage and pace mapping) to determine the locations of the ablation targets and (successful) ablation of the infarct-related VTs under the guidance of a CARTO 3D electroanatomical mapping system. Radiofrequency ablations were performed at the target regions during sinus rhythm. If nonclinical VT morphologies were inducible, those were also ablated; all the clinical ablation lesions executed during each procedure are shown in the corresponding figures. After completing ablation, programmed stimulation was repeated to determine inability to induce VT. Acute success was defined as the inability to induce clinical VT at the end of the procedure. 
The first human retrospective study involved 16 patients, all without ICDs (so that myocardial wall was not obscured in MRI) with the aim to compare the VAAT targets with the clinical lesions and determine whether the VAAT prediction fell within the areas ablated clinically. Eight of these patients were referred for catheter ablation of VT at the Johns Hopkins Hospital in the period between July 2006 and April 2013; the MRI data used in this study have been previously published ${ }^{23}$. The mean patient age in this retrospective study was $64.4 \pm 9$ years, the mean left ventricular ejection fraction (LVEF) was $38.4 \pm 12 \%$, and the cohort was $87.5 \%$ male. The other 8 patients were referred for catheter ablation of VT also at the Johns Hopkins Hospital in the period between November 2010 and October 2017; their MRI data have not been used for model reconstruction in any previous study. Results from 4 of these patients are shown in Fig. 2. The mean patient age in this group was $64.1 \pm 15.5$ years, the mean LVEF was $31.1 \% \pm 16.4 \%$, and the cohort was $75 \%$ male.

In the second retrospective study, 5 patients with ICDs were chosen randomly to demonstrate that VAAT could also be used to predict ablation targets in such patients; these patients were referred for catheter ablation of VT at the Johns Hopkins Hospital between December 2010 and November 2013. The mean patient age was 54.2 \pm 9.3 years, the mean LVEF was $39 \pm 13.8 \%$, and the cohort was $100 \%$ male. ICD artifact burden in the MRIs of these patients ranged from $0-62 \%$ of myocardial volume but did not cover the LGE regions (see main text).

For clarity, here we reproduce parts of the subsection on "EPS and ablation" from our earlier paper ${ }^{23}$. All patients underwent the standard EPS and ablation of scar-related VT under the 3D CARTO guidance during sinus rhythm. After ablation, programmed stimulation was repeated. Acute success was defined as the inability to induce clinical VT at the end of the procedure. If nonclinical VT morphologies were inducible, those were also ablated.

The Johns Hopkins Institutional Review Board (IRB) approved sharing of de-identified patient data with the modeling team for the retrospective study. Given the retrospective nature of the study, the IRB did not require patient informed consent.

\section{Prospective Patient Study}

The prospective study enrolled patients with infarct-related VT at the University of Utah and the University of Pennsylvania with the goal to demonstrate feasibility of using the noninvasive VAAT approach to guide clinical ablation. All patients had VT, and were referred for ablation; they all underwent LGE-MRI. At the University of Utah, three patients were targeted for ablation between January 2015 and December 2016. At the University of Pennsylvania, two patients were targeted for ablation between October and December 2017. The mean patient age was $73.5 \pm 3.4$ years, the mean LVEF was $33 \pm 4.4 \%$, and the cohort was $100 \%$ male.

At both clinical centers, during the ablation procedure VT was induced by programmed electrical stimulation. VAAT-predicted ablation site(s) were then imported in CARTO (see below for import and merger methodology detail). Ablation was then targeted to these sites using Irrigated Thermocool Ablation catheter. Post ablation, VT non-inducibility was verified by repeating programmed electrical stimulation. The protocol was approved by the 
Institutional Review Boards of the University of Utah Health Sciences Center and The Hospital of the University of Pennsylvania. All patients enrolled gave informed consent.

\section{VAAT Approach}

Heart structural model construction-Both animal and human models of 3D ventricular structure were reconstructed from the cardiac LGE-MRI data. The procedure is described in full detail in our recent publication on arrhythmia risk stratification in patients with myocardial infarction ${ }^{11}$. Briefly, for each heart, the myocardial boundaries in the MRI stack were contoured and the 3D ventricular wall geometry was reconstructed using a methodology based on variational implicit functions interpolation used previously by our team and validated with clinical data ${ }^{12}$. To represent the geometry of the infarct in each ventricular geometrical model, myocardial regions were classified as infarcted and noninfarcted areas by means of signal thresholding ${ }^{11}$. Each infarct region was further classified into scar and GZ using a full width half maximum approach validated previously ${ }^{24}$. The 3D geometries of the infarct zones were reconstructed and merged with the corresponding ventricular geometry reconstruction.

Construction of ventricular models from LGE-MRI scans of patients with ICDs involved additional processing steps to overcome the presence of artifact in the image. Following segmentation of the ventricular wall portion unobstructed by the shadow, we extrapolated the ventricular boundaries into the area occluded by the ICD artifact. Within the reconstructed ventricular wall, myocardium covered by the ICD shadow was delineated from unobstructed myocardium on the basis of the 3D radial distance from the ICD (Fig. 4, and Supplementary Figs. 4-5). Outside of the shadow, standard image processing classified the myocardium as non-infarcted, scar, or GZ, based on pixel intensity, as described above. The ventricular wall within the region of ICD shadow was assumed to have non-infarcted tissue properties, as it was outside of the zone of infarct. The burden of ICD artifact in the ventricular wall in each of the 5 patients is presented in Supplementary Table 3.

\section{Generating the computational meshes and assigning fiber orientation-For}

both animal and human heart models, finite-element ventricular meshes were generated, as described previously ${ }^{11,25,26}$, with an average resolution of $350 \mu \mathrm{m}$; ventricular models thus comprised $\sim 4$ million nodes. The choice of finite element size was dictated by the need to resolve wavefront propagation in the simulations while simultaneously minimizing computational expense ${ }^{11,27,28}$.

Finally, fiber orientations, specific to the individual geometry of the ventricles, were assigned to each ventricular computational mesh on a per-element basis, as done in our previous publications, ${ }^{11,26,29}$ using an efficient rule-based approach that we developed and validated $^{13}$. This fiber orientation methodology uses the Laplace-Dirichlet method to define transmural and apico-basal directions at every point in the patient-specific ventricles. It then employs bi-directional spherical linear interpolation to assign fiber orientations based on a set of fiber orientation properties (rules). After fiber orientation was assigned to the elements in the ventricular mesh, the corresponding "masks" of infarct scar and GZ were superimposed. Additional detail is presented in our recent publication ${ }^{11}$. 
After fiber orientations were assigned, the corresponding GZ and scar 'masks' are superimposed. GZ fiber orientations did not incorporate any changes in fiber orientation in that region (although there are changes in GZ conductivity and anisotropy; see below). The justification of this representation is based on a recent study ${ }^{30}$ that used sub-millimeterresolution diffusion tensor (DT) and LGE-MRI on a clinical scanner to examine the detailed organization of the infarct structure in the ventricles. The study demonstrated preservation of primary eigenvector (fiber) orientation at the thinned region of infarct in both human and porcine hearts.

Altogether, reconstruction of each patient heart took up to 8 hours.

Electrophysiological modeling-Detail regarding electrophysiological modeling in the swine hearts can be found in our recent paper ${ }^{29}$. Detail on all aspects of human ventricular electrophysiological modeling in myocardial infraction is presented in our recent publication ${ }^{11}$. Briefly, for all heart models, both animal and human, once the 3D finiteelement ventricular mesh was generated, regionally-uniform cell and tissue electrophysiological properties were assigned to the three regions outlined in the virtual heart from the LGE-MRI scans: scar, GZ, and non-infarcted tissue. All finite elements that belonged to the scar region were considered electrically non-conductive. In the patientspecific heart models, finite elements that belonged to non-infarcted tissue and GZ were assigned human ventricular cell action potential dynamics ${ }^{31}$; a different action potential model was used in the animal study ${ }^{29}$. Modifications to the ionic model based on experimental recordings were implemented to represent electrophysiological remodeling in the $\mathrm{GZ}^{11,29}$. Overall, the GZ action potentials were characterized by a longer duration, decreased upstroke velocity, and decreased peak amplitude compared to those in the noninfarcted myocardium, similar to what has been previously reported ${ }^{32,33}$. Specifically, as described in the animal model study ${ }^{23}$, action potential remodeling in the GZ was implemented by decreasing, the original action potential parameters as follows: peak sodium current to $38 \%$, peak L-type calcium current to $31 \%$, and peak potassium currents $\mathrm{I}_{\mathrm{Kr}}$ and $\mathrm{I}_{\mathrm{Ks}}$ to 30 and $20 \%$, respectively. The human action potential model in the patient studies was similarly modified to represent electrophysiological remodeling in the GZ, based on experimental data, as described in our previous patient study ${ }^{11}: 62 \%$ reduction in peak sodium current, $69 \%$ reduction in L-type calcium current and a reduction of 70 and $80 \%$ in potassium currents $\mathrm{I}_{\mathrm{Kr}}$ and $\mathrm{I}_{\mathrm{Ks}}$, respectively.

Tissue properties representing animal or human ventricular cell-to-cell electrical communication were also assigned to the non-infarcted and GZ regions, as described previously ${ }^{11}$; the GZ region was characterized with a decrease in transverse conductivity to reflect connexin- 43 remodeling in the infarct border zone. Similar to the latter study, the values of the non-infarcted tissue conductivities used here were 0.255 and $0.0775 \mathrm{~S} / \mathrm{m}$ in the longitudinal and transverse directions, respectively.

Simulation of electrical activity and numerical aspects-The propagation of electrical activity in a virtual heart was simulated by solving, using the finite-element method, a reaction-diffusion partial differential equation, representing the spread of current in the ventricular myocardium, together with the ordinary differential and algebraic 
equations representing myocyte membrane dynamics at each node in the mesh ${ }^{27}$.

Simulations of electrical activity in the patient-specific heart models were executed in a monodomain representation of the myocardium using the software package CARP (Johns Hopkins University, University of Bordeaux, University of Graz) on a parallel computing system $^{27,34}$. The system of equations was solved with a time step of $25 \mu$ s. Full detail regarding the simulations of electrical activity in the heart models is found in our recent publication $^{11}$ as well as in a number of earlier publications from our team ${ }^{35-37}$.

VT induction protocol-Each individualized ventricular model was subjected to pacing from multiple bi-ventricular locations in an attempt to elicit reentrant arrhythmias, thus revealing the potential of the disease-remodeled ventricles to cause degeneration of electrical signal propagation into arrhythmic activity following premature beats that originate at different locations in the heart. The protocol used here is identical to that in VARP (Virtualheart Arrhythmia Risk Predictor) approach in our previous study ${ }^{11}$, with the exception of the larger number of pacing sites utilized here. Each virtual heart was paced from 26 uniformly distributed (basal, medial, apical, lateral, septal, posterior, and anterior) locations, 9 on the right ventricular endocardium, and 17 on the left ventricular (LV) endocardium, the latter one in each of the AHA LV segments ${ }^{38}$. The rationale for choosing a large number of pacing sites was based on clinical studies, which have shown a positive correlation between the number of pacing sites and inducibility of ventricular arrhythmia ${ }^{39,40}$. The distribution of pacing sites throughout the ventricles ensured that the protocol covered a large range of possibilities for potential sites at which ectopic foci could emerge and captured all the possible arrhythmias that could arise from the given infarct morphology, and not only the one that was clinically manifested. Further increase in the number of pacing sites did not uncover more unique VTs. All pacing sites were assigned in the model automatically using an approach described previously ${ }^{41}$.

The pacing pulse trains were the same as in our previous studies ${ }^{11,26,29}$ and consisted of 8 pacing stimuli (S1) at a cycle length of $600 \mathrm{~ms}$ for the human models and $300 \mathrm{~ms}$ for the swine models. A premature stimulus (S2) was delivered $250 \mathrm{~ms}$ after S1. If S2 did not result in the generation of reentrant arrhythmia, the S1-S2 interval was shortened, in $10 \mathrm{~ms}$ steps, until arrhythmia was induced or the S2 failed to capture the tissue. If arrhythmia was not induced, an additional S3, and if necessary S4, were delivered in the same fashion as S2 (initially delivered $250 \mathrm{~ms}$ after previous stimulus and then shortened until arrhythmia was induced or the stimulus failed to capture). In all simulations, the size of the pacing electrode was $1 \times 1 \times 1 \mathrm{~mm}$, injecting transmembrane current. Simulations were monitored to ensure that in each case an excitation wave was initiated and propagated away from the pacing location.

To ensure computational tractability of the study, each simulation run calculated $\sim \mathrm{s}$ of electrical activity in the ventricles (corresponding to about 7 hours execution time), the first $5 \mathrm{~s}$ of which was the pacing protocol, and the remaining $2 \mathrm{~s}$ represented the post-pacing period used to detect the presence of arrhythmia. Arrhythmias were considered persistent if they did not self-terminate over the 2-s period. Initial simulations analyzing $5 \mathrm{~s}$ post-pacing activity in heart models demonstrated that arrhythmias that persisted for $2 \mathrm{~s}$ did not selfterminate after another $3 \mathrm{~s}$. This behavior is consistent with the deterministic nature of the 
model; the $7 \mathrm{~s}$ of total simulated activity also ensures turnaround time of $<24 \mathrm{~h}$ per patient in the prospective human study.

Individual reentrant arrhythmia periods in the simulations here could not be compared to those of the reentrant circuits induced during mapping in the human retrospective studies since the pacing sites in the virtual heart were at locations different from those of the clinical pacing sites. Importantly, simulations preliminary to this study demonstrated that changes in human action potential duration and conduction velocity within physiological boundaries changed the cycle length of the reentrant arrhythmia in a given heart model, however, the location of the organizing center of the arrhythmia (often isthmus or GZ-anchored phase singularity ${ }^{26}$ ) remained the same or nearly the same since it was predominantly determined by the individual distribution of scar and GZ. Indeed, for patient-specific models with structural remodeling, in simulations with $\pm 20 \%$ of the normal magnitude of the potassium current $\mathrm{I}_{\mathrm{K} 1}$ or the calcium current $\mathrm{I}_{\mathrm{CaL}}$ (resulting in action potential duration [APD] variation of $\pm 25 \mathrm{~ms}$ ) and $\pm 30 \%$ variation in tissue conductivity (resulting in conduction velocity variation of $\pm 10 \mathrm{~cm} / \mathrm{s}$ ), we found that the exact locations of the phase singularities varied only by $\unlhd .2 \mathrm{~cm}$ on average. These findings underscore the feasibility of ablation target prediction using patient-specific myocardial infarcted (MI) heart models reconstructed from patient LGE-MRI scans that incorporate average human electrophysiological properties (and thus do not require invasive electrophysiological measurements in the given patient for model parameter input).

\section{Validation of the electrophysiological modeling of pacing-induced} arrhythmias in the post-infarction ventricles-The approach used here to construct a model of the post-infarction ventricles by thresholding the infarct into scar and (homogeneous) GZ has been recently validated with experimental data. In a previous study, ${ }^{29}$ sock epicardial electrograms from infarct-related VT, obtained from in vivo swine hearts, were used to demonstrate that ventricular models reconstructed from MRI data of the corresponding hearts were able to predict fairly accurately the morphology of each VT reentrant circuit and its organizing center (e.g. isthmus). These results indicated that small heterogeneities in GZ, the Purkinje system, and additional regional electrophysiological heterogeneities play a secondary role in determining inducibility and organization of VT; primary influences include the geometrical morphologies of the scar and GZ, as well as the representation of different electrophysiological properties in non-infarcted tissue and GZ. These findings are consistent with those published previously ${ }^{26}$, which described a parameter sensitivity analysis of the GZ model representation. That study found that the inclusion of small scar heterogeneities with density within physiological range did not alter inducibility of infarct-related VT. These studies provided the initial justification for the electrophysiological modeling approach in this study.

The virtual-heart approach used here was recently validated in a human retrospective study ${ }^{11}$ of 41 post-infarction patients who underwent ICD implantation. Patients were followed for the primary endpoint of appropriate ICD firing due to ventricular arrhythmia or cardiac death for $4.8 \pm 2.9$ years. Blind to the clinical outcome, the virtual-heart approach assessed sudden cardiac death risk by determining VT inducibility in the patient-specific heart models following pacing from sites distributed throughout the left and right ventricles. The virtual 
heart test significantly outperformed several existing clinical metrics in predicting future arrhythmic events, validating the predictive capability of the modeling approach employed in the present study.

Finally, we are currently conducting an additional validation of our patient-specific modeling methodology in an ongoing clinical trial in patients receiving an ICD. An abstract of this study has been already published ${ }^{42}$.

\section{Approach to automatically determine the optimal VT ablation targets-We}

developed an approach to automatically identify, based on the simulated reentrant circuits, the optimal ablation targets for each VT morphology. The methodology was recently described and validated in patient-specific models of atypical left atrial flutter (LAFL) ${ }^{14}$, including comparisons with clinical ablation lesions that terminated LAFL. For clarity, below we reproduce parts of the subsection on "Identification and ablation of minimum cuts in reentrant wave propagation" from our earlier paper ${ }^{14}$. In this approach, we represent wave propagation during each cycle of reentry as a flow network. A flow network is a mathematical graph that abstracts directional movement between interconnected objects. Interconnected objects are represented as vertices in a graph; links between adjacent pairs of vertices, where flow can pass through, are called edges; and the maximum possible flow between two adjacent vertices is termed edge capacity.

In this study, each vertex in the flow network corresponded to an element in the LGE-MRIderived geometric model. Two vertices in the network were defined to be adjacent to each other if they (1) corresponded to elements that shared a common face and (2) if the difference in activation time between these elements was $<20 \mathrm{~ms}$. An edge in the flow network corresponded to the shared face between adjacent vertices. The capacity of a network edge was defined to be equal to the cross-sectional area of the corresponding face.

The "minimum cut" (MC) in a flow network represents the number of edges that, when removed, separate the 3D network into 2 disconnected components. Here it was determined using the Boykov-Kolmogorov algorithm ${ }^{43}$. The tissue that comprises each minimum cut represents the in silico equivalent of the minimal set of ablation lesions needed to terminate VT. In silico ablation was performed by rendering tissue within $1 \mathrm{~mm}$ of the MC nonexcitable. The VT induction protocol was then repeated to establish that VT was no longer inducible. If a new VT arose after implementing the calculated ablation lesions, then additional targets corresponding to that VT were calculated. The protocol was repeated until VT was no longer inducible.

Examples that illustrate the advantages of the MC approach, highlighting its 3D nature, can be found in our earlier published study ${ }^{14}$ (see the Online Supplementary Materials in that paper). Importantly, the use of the MC algorithm bypasses the need to establish in each patient what constitutes an ablation target, whether channels in the scar ${ }^{17,18}$, part of or the entire $\mathrm{GZ}^{19}$, or reentrant drivers ${ }^{20}$. Finally, as already discussed in the main text, since the predicted targets are $3 \mathrm{D}$, in a given patient case they may not be reachable via a particular endo- or epicardial approach; in such cases, we envision the targets to be re-calculated, 
taking into account that a given portion of the initial predicted target will need to remain excitable.

\section{Merger of VAAT predicted targets with CARTO electroanatomical map in the} prospective patient studies-In order to guide the ablation using the VAAT approach, the predicted targets needed to be imported into the CARTO electroanatomical mapping system (CARTO 3, BioSense Webster, Diamond Bar, CA) during the clinical procedure. To do so, we extracted the predicted targets from the virtual heart model on surface meshes and exported them into CARTO as Virtualization Toolkit (VTK) files (Kitware Inc.), ensuring that the resulting files are compatible with the CARTO system. To register the VAAT targets to the patient heart during the clinical procedure, additional landmarks from the patient's virtual heart geometric model were required for the registration process; the latter was performed with CartoMerge within the electroanatomical navigation system. The following surfaces were also extracted from the patient's virtual heart model: left ventricular endocardium, left ventricular apex, right ventricular endocardium, epicardium, and infarct surfaces; these all served as landmarks. Additional surfaces segmented and used as landmarks included the aorta, the left aortic cusp, and the right aortic cusp. All surfaces were exported as VTK files and imported into the CARTO system at the beginning of the clinical ablation procedure. The registration process performed with CartoMerge aligned the coordinates of the left ventricular apex and left and right aortic cusps of the model landmarks to the coordinates of the left ventricular apex and left and right aortic cusps of the surfaces created with the intra-cardiac ultrasound catheter during the procedure. This landmark-based registration superimposed the virtual heart model landmarks, including the VAAT targets, to those of the patient heart. This enabled the clinician to navigate the ablation catheter to the VAAT targets accurately. After the procedure, the CARTO study, which included the CARTO clinical electroanatomical surfaces and the ablation lesion locations registered to the patient's virtual heart model were exported for analysis. The exported files were converted to VTK files, and Paraview (Kitware Inc.) was used for visualization and comparison of the VAAT targets with the clinical ones.

The mean and standard deviation of the distance between the registered CARTO surface and the model LV endocardium was $5.77+/-4.23 \mathrm{~mm}$.

\section{Uncertainty in Target Predictions and limitations of the VAAT approach}

The VAAT strategy involves the use of personalized geometry and distribution of structural remodeling, but it is not personalized with respect to the electrophysiology of the patient. We use the same pre-determined "average" electrophysiology modelling to represent noninfarcted tissue and GZ in all of our patient-derived models. An ideal option would be to use personalized electrophysiology in each model, however acquisition of such personalized information would most likely be invasive, in contrast to the VAAT approach, which offers a non-invasive prediction of the ablation targets. Thus, the VAAT-predicted ablation targets have a level of uncertainty associated with the fact that pre-determined electrophysiology is used. One could potentially endeavor to re-calculate, as part of the VAAT workflow, the predicted patient-specific ablation targets with different pre-determined electrophysiology and then examine the differences in the resulting targets, if any. However, this is currently 
impossible within the clinical workflow, because of the short time window to conduct the simulations, that between clinical LGE-MRI acquisition and the ablation procedure; hospital workflow necessitates cardiac MRI acquisition the day before procedure. The issue is further exacerbated by the fact that as part of VAAT, in-silico ablation with the predicted targets is also conducted and the model paced again from all pacing sites to examine VT inducibility this procedure is repeated until the substrate is non-inducible for VT. Thus, to be able to predict the VT ablation targets non-invasively and as part of the clinical workflow, the use of pre-determined electrophysiology, with its potential uncertainty (different for each patient), will need to be retained.

In VAAT, this potential uncertainty is compounded with that associated with co-registration of predicted targets and CARTO images, as described in the previous section. Other limitations of the approach include the fact that clinical LGE-MRI quality could be operatordependent and thus could include uncertainty in image processing of scar and GZ. Despite the presence of some level of uncertainty, determining the ablation targets non-invasively using VAAT is shown here to be a very promising approach. Most likely, VAAT will not succeed in all patients, but we are expecting it to be able to significantly raise the success rate in infarct-related VT ablation. This is similar to our approach to sudden cardiac death risk stratification, VARP ${ }^{11}$ while we did not correctly predict every patient's clinical outcome, the VARP prediction was significantly (by a wide margin) better than any clinical indices used before. Overall, should VAAT be successfully tested in larger prospective studies, its adoption is expected to result in significant clinical benefits: 1) avoiding extensive invasive mapping to determine the ablation targets, 2) decreasing potential complications due to the short duration of the procedure, 3 ) a more accurate and 3D set of targets (rather than those determined from a surface interrogation) resulting in smaller ablation lesions, and 4) a decrease in repeated procedures, as VAAT targets incorporate those that would emerge after initial ablation.

\section{Code Availability}

The image processing software CardioViz3D can be freely obtained from http://wwwsop.inria.fr/asclepios/software/CardioViz3D. The open-source software Seg3D used in graylevel thresholding can be obtained from http://www.sci.utah.edu/cibc-software/seg3d.html. Computational meshes are generated using the software Simpleware ScanIP, available from Synopsys. The ionic models are freely available from the repository CellML (https:// www.cellml.org). Regarding the rule-based approach to assign fiber orientations in the computational mesh, the original publication ${ }^{13}$ presents a set of algorithms and subroutines that can be easily implemented. The electrophysiology simulator CARP has been developed by our team and used in over 60 publications; it can currently be obtained from Johns Hopkins University, the University of Bordeaux, or the University of Graz. The ventricular simulations can also be executed using the open-source software CHASTE: http:// www.cs.ox.ac.uk/chaste.

\section{Data Availability}

The authors declare that all data supporting the findings of this study are available within the paper and its supplementary information. The patient MRI images used to construct the 
personalized heart models are available on request and on approval of Johns Hopkins Institutional Review Board. Source data for activation maps shown in Figs. 1-4 are available in figshare, with the identifier doi:10.6084/m9.figshare.6613289 (ref. 44).

\section{Supplementary Material}

Refer to Web version on PubMed Central for supplementary material.

\section{Acknowledgements.}

This work was supported by NIH Pioneer Award (DP1-HL123271) to NAT.

\section{References}

1. Stevenson WG et al. Radiofrequency catheter ablation of ventricular tachycardia after myocardial infarction. Circulation 98, 308-314 (1998). [PubMed: 9711935]

2. Aliot EM et al. EHRA/HRS Expert Consensus on Catheter Ablation of Ventricular Arrhythmias. Heart Rhythm 6, 886-933, 10.1016/j.hrthm.2009.04.030 (2009). [PubMed: 19467519]

3. Zhong H, Lacomis JM \& Schwartzman D On the accuracy of CartoMerge for guiding posterior left atrial ablation in man. Heart Rhythm 4, 595-602, 10.1016/j.hrthm.2007.01.033 (2007). [PubMed: 17467627]

4. Brugada $\mathbf{J}$ et al. Nonsurgical transthoracic epicardial radiofrequency ablation: an alternative in incessant ventricular tachycardia. J Am Coll Cardiol 41, 2036-2043 (2003). [PubMed: 12798578]

5. Sosa E, Scanavacca M, d'Avila A, Oliveira F \& Ramires JA Nonsurgical transthoracic epicardial catheter ablation to treat recurrent ventricular tachycardia occurring late after myocardial infarction. J Am Coll Cardiol 35, 1442-1449 (2000). [PubMed: 10807445]

6. Dong $\mathrm{J}$ et al. Impact of heart rhythm status on registration accuracy of the left atrium for catheter ablation of atrial fibrillation. J Cardiovasc Electrophysiol 18, 1269-1276, 10.1111/j. 1540-8167.2007.00956.x (2007). [PubMed: 17850289]

7. de Bakker JM et al. Reentry as a cause of ventricular tachycardia in patients with chronic ischemic heart disease: electrophysiologic and anatomic correlation. Circulation 77, 589-606 (1988). [PubMed: 3342490]

8. Peters NS \& Wit AL Myocardial architecture and ventricular arrhythmogenesis. Circulation 97, 1746-1754 (1998). [PubMed: 9591770]

9. Callans DJ et al. Efficacy of radiofrequency catheter ablation for ventricular tachycardia in healed myocardial infarction. Am J Cardiol 82, 429-432 (1998). [PubMed: 9723628]

10. Calkins $\mathrm{H}$ et al. Catheter ablation of ventricular tachycardia in patients with structural heart disease using cooled radiofrequency energy: results of a prospective multicenter study. Cooled RF Multi Center Investigators Group. J Am Coll Cardiol 35, 1905-1914 (2000). [PubMed: 10841242]

11. Arevalo $\mathrm{HJ}$ et al. Arrhythmia risk stratification of patients after myocardial infarction using personalized heart models. Nat Commun 7, 11437, 10.1038/ncomms11437 (2016). [PubMed: 27164184]

12. Prakosa A et al. Methodology for image-based reconstruction of ventricular geometry for patientspecific modeling of cardiac electrophysiology. Prog Biophys Mol Biol 115, 226-234, 10.1016/ j.pbiomolbio.2014.08.009 (2014). [PubMed: 25148771]

13. Bayer JD, Blake RC, Plank G \& Trayanova NA A novel rule-based algorithm for assigning myocardial fiber orientation to computational heart models. Ann Biomed Eng 40, 2243-2254, 10.1007/s10439-012-0593-5 (2012). [PubMed: 22648575]

14. Zahid $\mathrm{S}$ et al. Feasibility of using patient-specific models and the "minimum cut" algorithm to predict optimal ablation targets for left atrial flutter. Heart Rhythm 13, 1687-1698, 10.1016/ j.hrthm.2016.04.009 (2016). [PubMed: 27108938] 
15. Lardo AC et al. Visualization and temporal/spatial characterization of cardiac radiofrequency ablation lesions using magnetic resonance imaging. Circulation 102, 698-705 (2000). [PubMed: 10931812]

16. Tung R, Josephson ME, Reddy V, Reynolds MR \& Investigators S-V Influence of clinical and procedural predictors on ventricular tachycardia ablation outcomes: an analysis from the substrate mapping and ablation in Sinus Rhythm to Halt Ventricular Tachycardia Trial (SMASH-VT). J Cardiovasc Electrophysiol 21, 799-803, 10.1111/j.1540-8167.2009.01705.x (2010). [PubMed: 20132389]

17. Hsia HH, Lin D, Sauer WH, Callans DJ \& Marchlinski FE Anatomic characterization of endocardial substrate for hemodynamically stable reentrant ventricular tachycardia: identification of endocardial conducting channels. Heart Rhythm 3, 503-512, 10.1016/j.hrthm.2006.01.015 (2006). [PubMed: 16648052]

18. Tung R et al. Impact of local ablation on interconnected channels within ventricular scar: mechanistic implications for substrate modification. Circ Arrhythm Electrophysiol 6, 1131-1138, 10.1161/CIRCEP.113.000867 (2013). [PubMed: 24162832]

19. Verma A et al. Relationship between successful ablation sites and the scar border zone defined by substrate mapping for ventricular tachycardia post-myocardial infarction. J Cardiovasc Electrophysiol 16, 465-471, 10.1046/j.1540-8167.2005.40443.x (2005). [PubMed: 15877614]

20. Krummen DE et al. Modifying Ventricular Fibrillation by Targeted Rotor Substrate Ablation: Proof-of-Concept from Experimental Studies to Clinical VF. J Cardiovasc Electrophysiol 26, 1117-1126, 10.1111/jce.12753 (2015). [PubMed: 26179310]

21. Nikolov P, Prakosa A, Arevalo HJ, Wu KC \& Trayanova N A Novel Approach to Arrhythmia Risk Stratification in Patients With Non-ischemic Cardiomyopathy. Circulation 134, A20903-A20903 (2016).

22. Estner HL et al. The critical isthmus sites of ischemic ventricular tachycardia are in zones of tissue heterogeneity, visualized by magnetic resonance imaging. Heart Rhythm 8, 1942-1949, 10.1016/ j.hrthm.2011.07.027 (2011). [PubMed: 21798226]

23. Ashikaga $\mathrm{H}$ et al. Feasibility of image-based simulation to estimate ablation target in human ventricular arrhythmia. Heart Rhythm 10, 1109-1116, 10.1016/j.hrthm.2013.04.015 (2013). [PubMed: 23608593]

24. Schmidt A et al. Infarct tissue heterogeneity by magnetic resonance imaging identifies enhanced cardiac arrhythmia susceptibility in patients with left ventricular dysfunction. Circulation 115 , 2006-2014, 10.1161/CIRCULATIONAHA.106.653568 (2007). [PubMed: 17389270]

25. Prassl AJ et al. Automatically generated, anatomically accurate meshes for cardiac electrophysiology problems. IEEE Trans Biomed Eng 56, 1318-1330, 10.1109/TBME. 2009.2014243 (2009). [PubMed: 19203877]

26. Arevalo H, Plank G, Helm P, Halperin H \& Trayanova N Tachycardia in post-infarction hearts: insights from 3D image-based ventricular models. PLoS One 8, e68872, 10.1371/journal.pone. 0068872 (2013). [PubMed: 23844245]

27. Plank $\mathrm{G}$ et al. From mitochondrial ion channels to arrhythmias in the heart: computational techniques to bridge the spatio-temporal scales. Philos Trans A Math Phys Eng Sci 366, 33813409, 10.1098/rsta.2008.0112 (2008). [PubMed: 18603526]

28. Gurev V, Lee T, Constantino J, Arevalo H \& Trayanova NA Models of cardiac electromechanics based on individual hearts imaging data: image-based electromechanical models of the heart. Biomech Model Mechanobiol 10, 295-306, 10.1007/s10237-010-0235-5 (2011). [PubMed: 20589408]

29. Deng D et al. Accuracy of prediction of infarct-related arrhythmic circuits from image-based models reconstructed from low and high resolution MRI. Front Physiol 6, 282, 10.3389/fphys. 2015.00282 (2015). [PubMed: 26528188]

30. Pashakhanloo F et al. Submillimeter diffusion tensor imaging and late gadolinium enhancement cardiovascular magnetic resonance of chronic myocardial infarction. J Cardiovasc Magn Reson 19, 9, 10.1186/s12968-016-0317-3 (2017). [PubMed: 28122618] 
31. ten Tusscher KH, Noble D, Noble PJ \& Panfilov AV A model for human ventricular tissue. Am J Physiol Heart Circ Physiol 286, H1573-1589, 10.1152/ajpheart.00794.2003 (2004). [PubMed: 14656705]

32. Decker KF \& Rudy Y Ionic mechanisms of electrophysiological heterogeneity and conduction block in the infarct border zone. Am J Physiol Heart Circ Physiol 299, H1588-1597, 10.1152/ ajpheart.00362.2010 (2010). [PubMed: 20709867]

33. Cabo C \& Boyden PA Electrical remodeling of the epicardial border zone in the canine infarcted heart: a computational analysis. Am J Physiol Heart Circ Physiol 284, H372-384, 10.1152/ ajpheart.00512.2002 (2003). [PubMed: 12388240]

34. Vigmond EJ, Weber dos Santos R, Prassl AJ, Deo M \& Plank G Solvers for the cardiac bidomain equations. Prog Biophys Mol Biol 96, 3-18, 10.1016/j.pbiomolbio.2007.07.012 (2008). [PubMed: 17900668]

35. Rodriguez B, Li L, Eason JC, Efimov IR \& Trayanova NA Differences between left and right ventricular chamber geometry affect cardiac vulnerability to electric shocks. Circ Res 97, 168 175, 10.1161/01.RES.0000174429.00987.17 (2005). [PubMed: 15976315]

36. Rantner LJ et al. Three-dimensional mechanisms of increased vulnerability to electric shocks in myocardial infarction: altered virtual electrode polarizations and conduction delay in the periinfarct zone. J Physiol 590, 4537-4551, 10.1113/jphysiol.2012.229088 (2012). [PubMed: 22586222]

37. Bishop MJ et al. The role of photon scattering in optical signal distortion during arrhythmia and defibrillation. Biophys J 93, 3714-3726, 10.1529/biophysj.107.110981 (2007). [PubMed: 17978166]

38. Cerqueira MD et al. Standardized myocardial segmentation and nomenclature for tomographic imaging of the heart. A statement for healthcare professionals from the Cardiac Imaging Committee of the Council on Clinical Cardiology of the American Heart Association. Circulation 105, 539-542 (2002). [PubMed: 11815441]

39. Piccini JP et al. Mode of induction of ventricular tachycardia and prognosis in patients with coronary disease: the Multicenter UnSustained Tachycardia Trial (MUSTT). J Cardiovasc Electrophysiol 20, 850-855, 10.1111/j.1540-8167.2009.01469.x (2009). [PubMed: 19490266]

40. Buxton AE Programmed ventricular stimulation: not dead. Circulation 129, 831-833, 10.1161/ CIRCULATIONAHA.113.007747 (2014). [PubMed: 24381210]

41. Vadakkumpadan F, Trayanova N \& Wu KC Image-based left ventricular shape analysis for sudden cardiac death risk stratification. Heart Rhythm 11, 1693-1700, 10.1016/j.hrthm.2014.05.018 (2014). [PubMed: 24854217]

42. Ranjan R et al. Personalized MRI-Based Modeling Predicts Ventricular Tachycardia Vulnerability in Patients Receiving Primary Prevention ICDs. Circulation 134, A16247-A16247 (2016).

43. Boykov Y \& Kolmogorov V An experimental comparison of min-cut/max-flow algorithms for energy minimization in vision. IEEE Trans Pattern Anal Mach Intell 26, 1124-1137, 10.1109/ TPAMI.2004.60 (2004). [PubMed: 15742889]

44. Prakosa et al. Dataset for Personalized Virtual-heart Technology for Guiding the Ablation of Infarct-related Ventricular Tachycardia. figshare 10.6084/m9.figshare.6613289 (2018). 
a

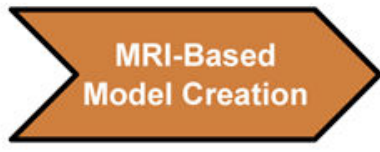

b
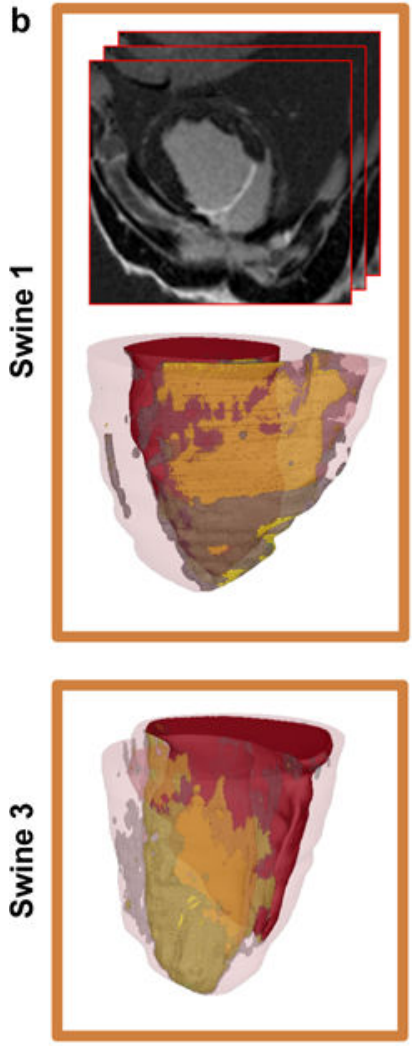

Non-Injured

Scar
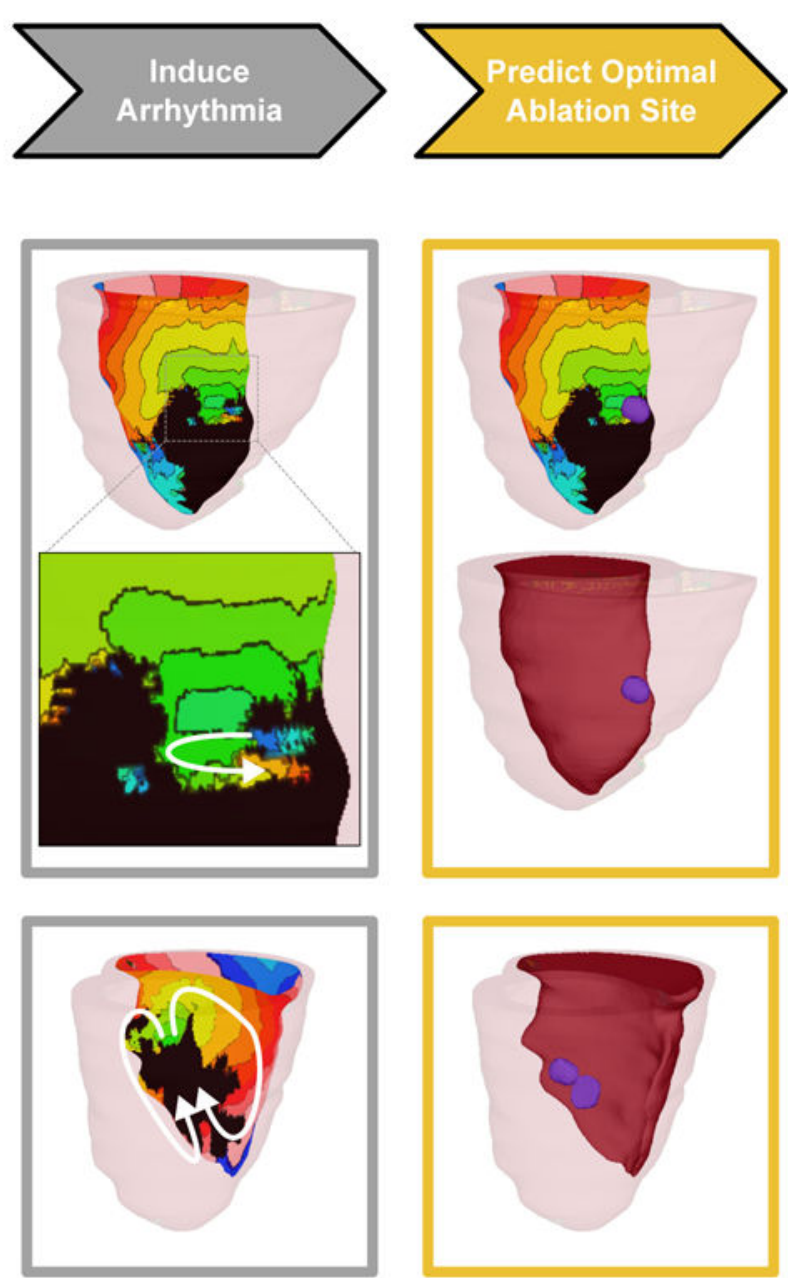

Gray Zone Ablation

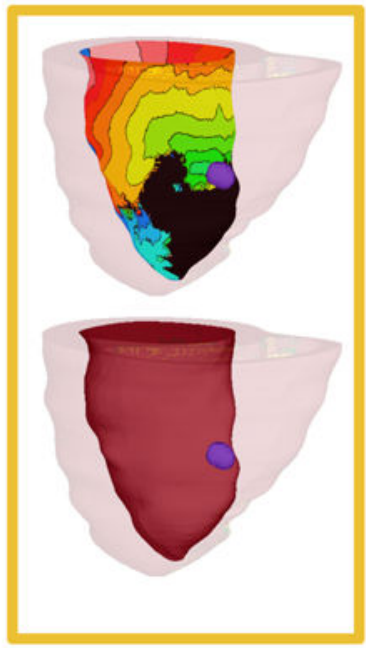

Retrospective

Validation of Prediction

Prospective Ablation in the Clinic
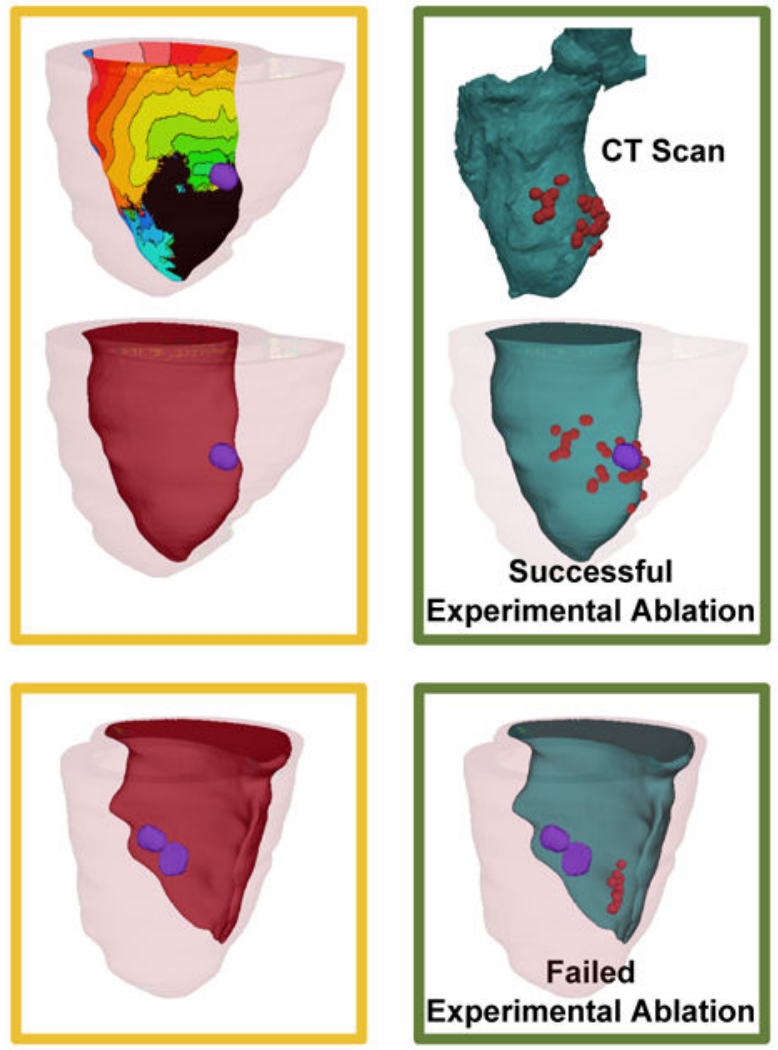

$0 \mathrm{~ms}$

Figure 1: Virtual-heart ablation-target (VAAT) prediction: protocol and results from the animal study.

(a) Flow-chart summarizing the protocol (arrowed steps) and retrospective and prospective studies. (b) In silico models and predictions for one case of successful mapping-guided ablation in Swine 1 (top), and one case of failed mapping-guided ablation in Swine 3 (bottom). Panels, from left to right: reconstructed ventricular model with different remodeled regions and the upper, top panel showing the LGE-MRI stack for Swine 1; endocardial electrical activation maps of the infarct-related VTs with white arrows showing the direction of propagation of the excitation wave, inset shows the zoomed-in propagation waves through channels in the scar in Swine 1; purple circles correspond to in silico predicted ablation targets on the ventricular endocardial surface; CARTO XP ventricular geometry from postablation CT scans co-registered with the MRI-based model for comparison of the predicted ablation targets with experimental mapping-based endocardial ablation locations, where red dots correspond to location of the tip of the catheter during ablation. Panel frame colors correspond to the protocol steps outline in Fig. 1a. Non-injured, scar, gray zone tissues and VAAT ablation targets are shown in red, yellow, gray, and purple respectively. Color scale 
indicates activation times, from earliest to latest, for images in column 2; black indicates tissue regions that did not activate. 


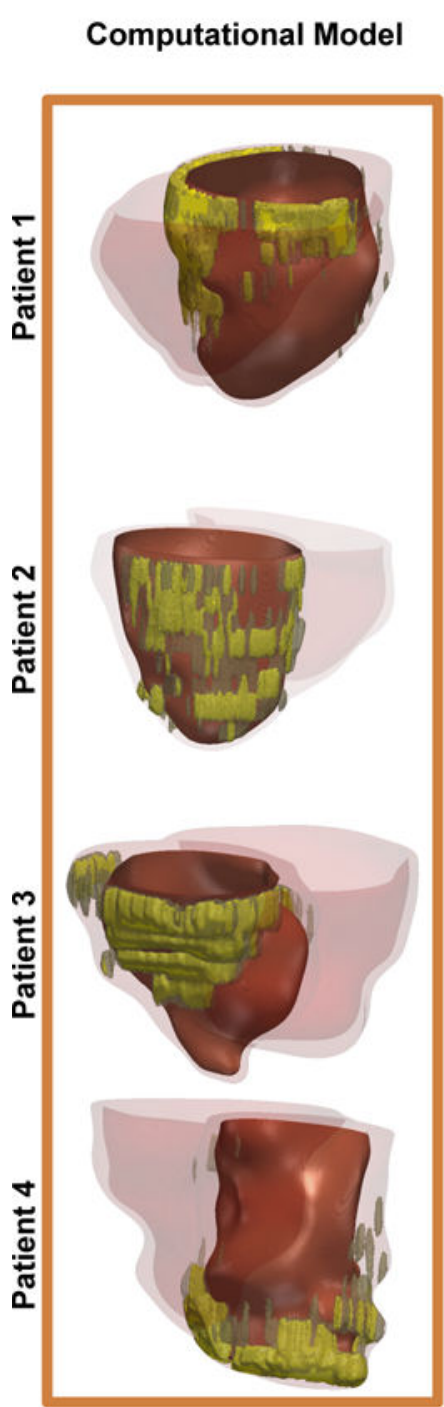

Non-Injured Scar
Induced Arrhythmia
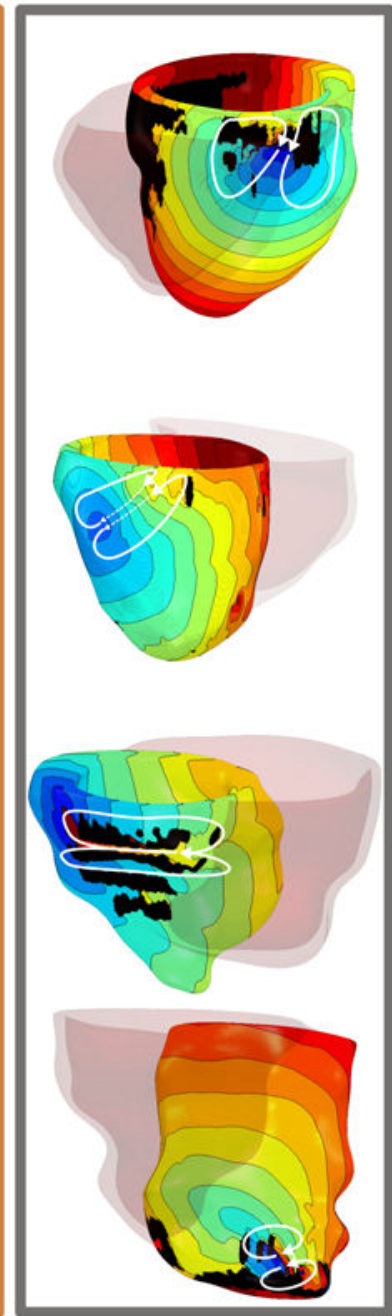

Gray Zone Ablation

\section{Predicted Optimal} Ablation Site
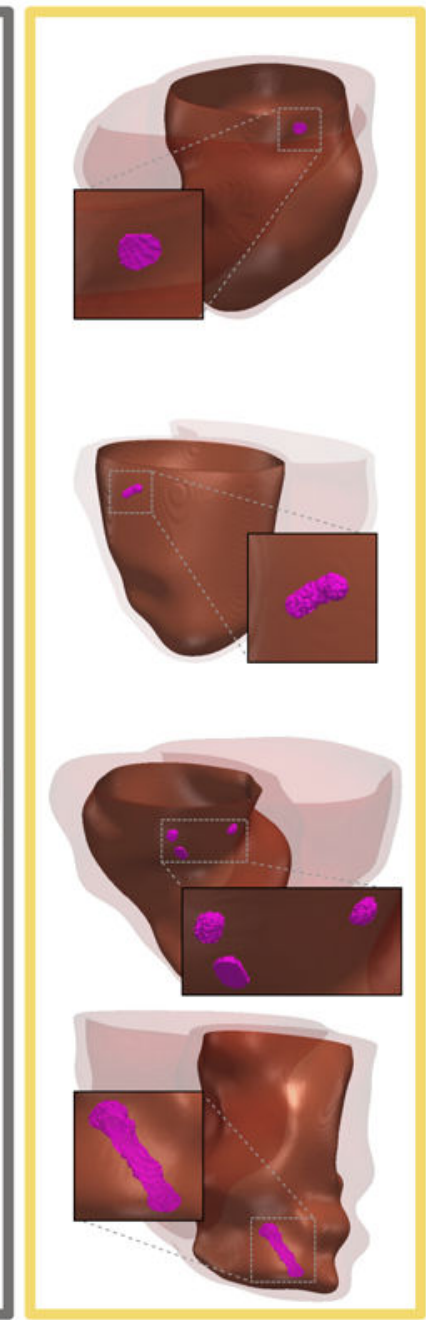

$0 \mathrm{~ms}$

\section{Comparison with Clinical Ablation}

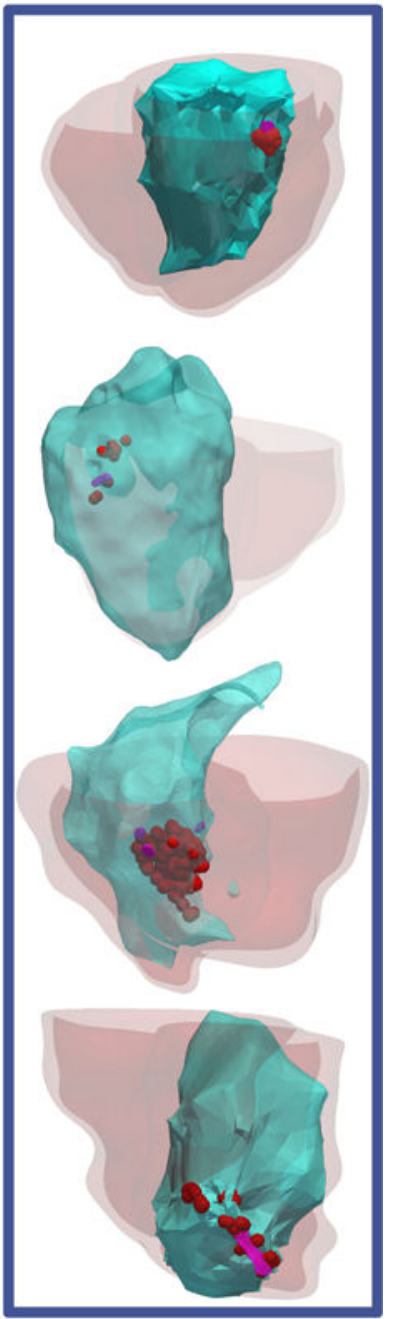

$450 \mathrm{~ms}$

Figure 2: Results from retrospective human study.

Representative examples of in silico models and predictions from 4 patients are shown (top to bottom). Left to right: reconstructed ventricular computational model with different structurally-remodeled regions; electrical activation maps of the infarct-related VTs after induced arrhythmia on the epi-or endocardial surfaces and white arrows showing the direction of propagation of the excitation wave; purple regions correspond to VAAT predicted ablation targets on the endocardial surface, insets show the zoomed-in VAAT predictions; and co-registration of VAAT targets with the CARTO 3 endocardial surface (green) showing clinical ablation where red dots correspond to locations of the tip of the catheter during ablation. Non-injured, scar, gray zone tissues and VAAT ablation targets are shown in red, yellow, gray, and purple respectively. Color scale indicates activation times, from earliest to latest, for images in column 2; black indicates tissue regions that did not activate. 


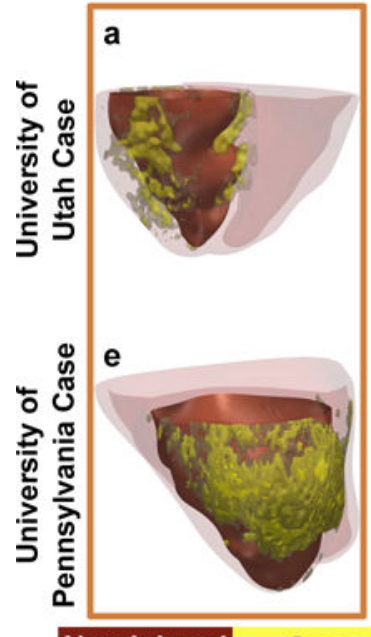

Non-Injured Scar
Predicted Optimal Ablation Site
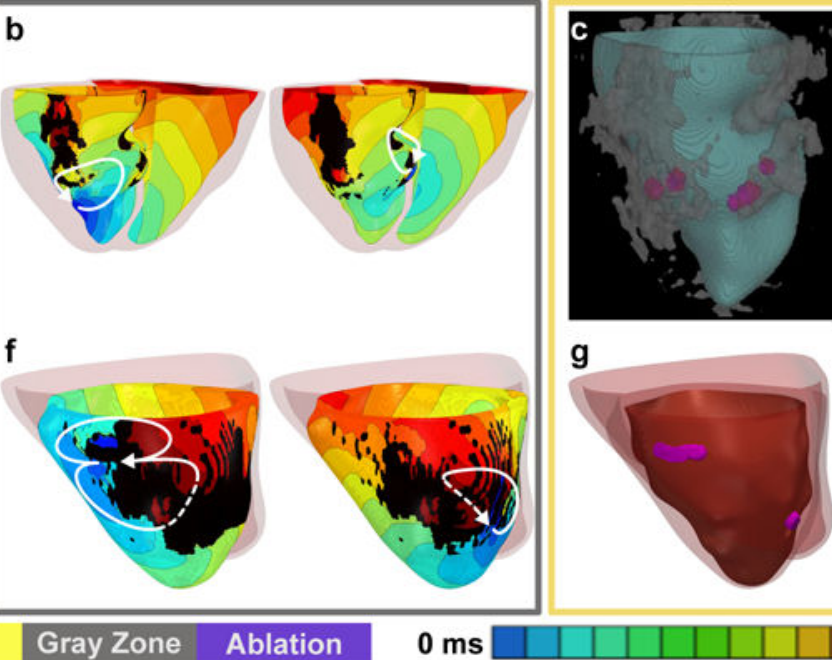

g

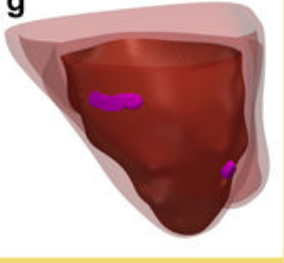

Comparison with Clinical Ablation

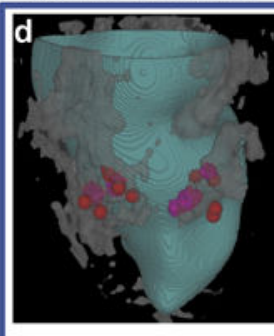

h

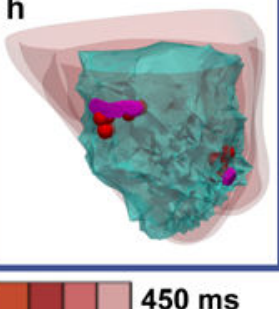

Figure 3: Results from the prospective human study.

VAAT-guided ablation (a-d) for one patient at the University of Utah; (e-h) and at the University of Pennsylvania. (a,e) Reconstructed ventricular models with different remodeled regions, $(b, f)$ Activation maps corresponding to the two VT morphologies induced in the Utah and Pennsylvania patient model respectively with white arrows depicting the direction of propagation of the excitation wave, $(c, g)$ VAAT-predicted ablation targets for the two VT morphologies, $(\mathrm{d}, \mathrm{h})$ Co-registration of the VAAT-predicted targets (purple) with the CARTO 3 endocardial surface (green). The red dots correspond to locations of the tip of the catheter during ablation. The left ventricular endocardial surface is shown in green and the total infarct region is shown in gray (gray zone). Non-injured and scar tissues are shown in red and yellow respectively. Color scale indicates activation times, from earliest to latest, for images in column 2; black indicates tissue regions that did not activate. 
LGE-MRI and Model

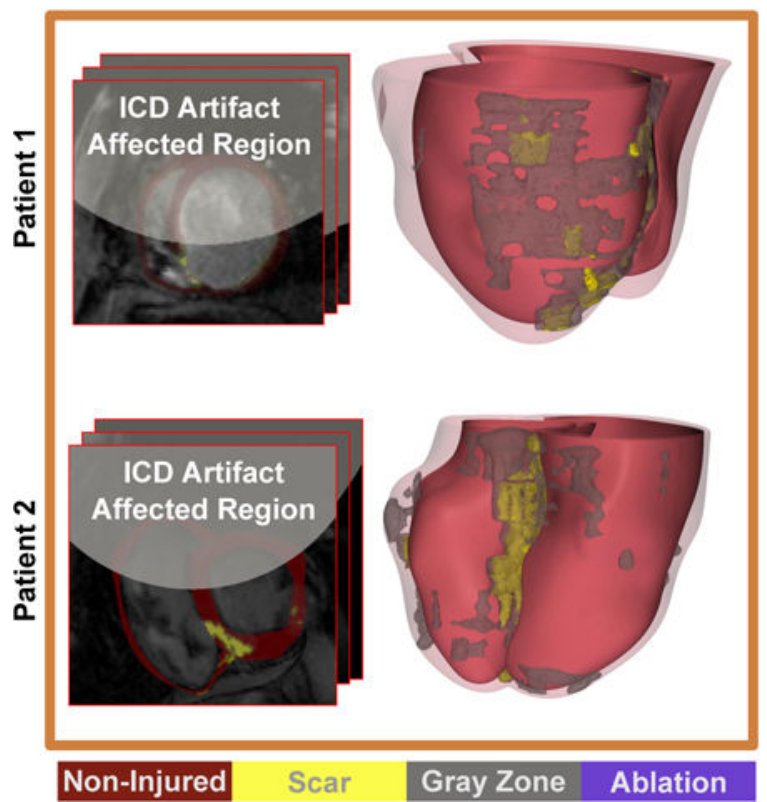

Induced Arrhythmia

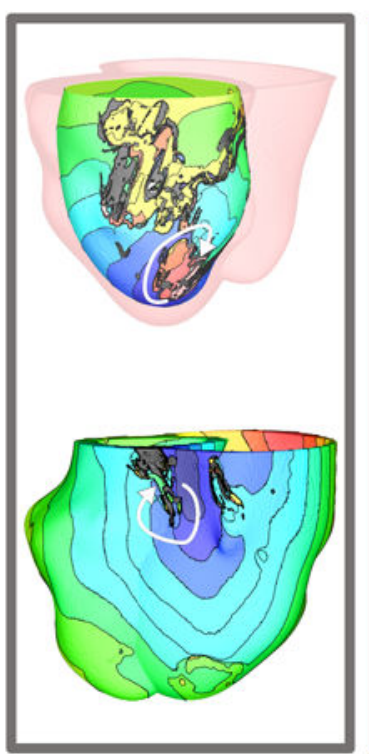

$0 \mathrm{~ms}$
Comparison With Clinical Ablation

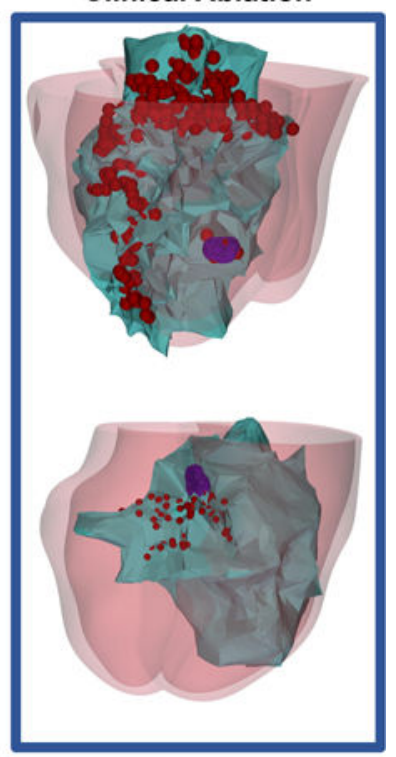

Figure 4: Results from the retrospective human study in patients with ICDs.

Representative examples of in silico models and predictions from 2 patients are shown (top and bottom). The myocardial wall artifact burden was $59 \%$ and $46 \%$ for patient 1 and 2 respectively. Left panel: LGE MRI scans with ICD artifact burden and reconstructed ventricular models with different remodeled regions; middle panel: electrical activation maps of the infarct-related VTs on the epi-or endocardial surfaces (chosen for best visualization) and white arrows showing direction of VT propagation; right panel: and coregistration of ventricular model surfaces and the VAAT ablation targets (purple) with the CARTO endocardial surfaces (green) showing clinical ablation locations corresponding to red dots representing locations of the tip of the catheter during ablation. The CARTO endocardial surfaces (green surfaces, right panel) show the left ventricle for patient 1 and the right ventricle for patient 2 co-registered with the corresponding MRI shells obtained in the corresponding individuals at the time of their clinical procedure. In Patient 1 , the predicted ablation target overlapped with the clinical at the same location. The clinical ablation also identified extensive lesions at the periphery of the infarct (the gray zone). In patient 2, the VAAT lesion was within the area ablated clinically. Non-injured, scar, gray zone tissues and VAAT ablation targets are shown in red, yellow, gray, and purple respectively. Color scale indicates activation times, from earliest to latest, for images in column 3; black indicates tissue regions that did not activate. 